\title{
Emergency Resource Scheduling During COVID-19
}

\author{
Lingfang $\mathrm{Wu}^{1}$, Xiaolin Xiang*2 \\ ${ }^{1}$ Business school, Sichuan University,Chengdu, Sichuan, 610065, China \\ ${ }^{2}$ Business school, Sichuan University,Chengdu, Sichuan, 610065, China
}

\begin{abstract}
Due to the suddenness and uncertainty of the COVID-19, sufficient emergency resources should be allocated to the appropriate emergency space within a limited time. In order to ensure the smooth implementation of emergency resource scheduling, emergency resource must be timely and effectively supplied. Therefore, it is of great significance to study how to reasonably distribute emergency resource and how to optimize the vehicle routing problem to improve the efficiency of emergency. Based on the research of capacitated vehicle routing problem (CVRP) solved by traditional genetic algorithm, this paper proposes simulated annealing-genetic algorithm, which has been verified to effectively solve the capacitated vehicle routing problem.
\end{abstract}

\section{Introduction}

In December 2019, the COVID-19 broke out in Wuhan, Hubei Province, and the scale of the epidemic increased continuously. As a severe disaster area, Wuhan is facing the problem of lack of medical materials (masks, protective glasses). Therefore, the emergency resource scheduling has become an important issue worthy of our attention and research. Due to the urgent need of emergency resource, scientific and efficient improvement of scheduling, reducing the total dispatch distance have become the main research issues. Among them, emergency vehicle scheduling is the key to solve the problem. At present, more and more scholars study the optimization of capacitated vehicle routing problem from Different levels. In the research of emergency vehicle scheduling problem, Chen Gang, Zhang Jin et al. [1] established a two-level optimization model including vehicle routing and emergency resource allocation, forming a three-level agile distribution system of reserve, distribution center and disaster site. earthquake data is used as the algorithm simulation experimental data to verify the practicability and effectiveness of the model and the improved ant colony algorithm. Chen Gangtie, Shuai Bin [2] established a connection between fuzzy network and time, and used the shortest total dispatch time as the objective function of the problem to solve and optimize the emergency vehicle scheduling problem. Chen Sen, Jiang Jiang et al. [3] Comprehensively considering the implementation of the road network structure and establishing a joint optimization model for the problem, an improved genetic algorithm solution example is proposed to verify the feasibility and practicability of the problem model and the improved genetic algorithm. Yang Bin, Wen Hongrui et al. [4] established a bi-level programming model based on Bayesian dynamic, The upper model aims at minimizing the cost of emergency resource distribution, while the lower model describes the improvement status of different disaster points, then Bayesian combined with particle swarm optimization algorithm is used to solve this bi-level programming. Li Shuanglin et al. [5] established a logistics distribution model of multi-objective location multimodal transportation, and used multi-objective genetic algorithm to solve the problem, so that the total time of emergency resource distribution is the shortest, and the resource demand satisfaction rate of disaster area is the highest. Stanley et al. [6] studied the formation of clusters from different starting angles In the Sweep variant, and used velocity tentative particle swarms to optimize the routh, which can finally solve the CVRP problem better.

Based on the analysis of relevant literature, this paper describes the CVRP in the process of emergency resource scheduling, and analyzes that the influencing factors of the process are vehicle capacity limit and distribution space distance, constructs a CVRP model suitable for emergency resource scheduling process, and designs a simulated annealing-genetic algorithm (SA-GA). Finally, the feasibility of the model and algorithm is proved by algorithm simulation.

\section{Problem Description And Model Establishment}

\subsection{Scenario description}

${ }^{*}$ Corresponding author's e-mail: scugsxxl@sina.com 
As a severe disaster area of the COVID-19, there is an urgent need for emergency supplies in Wuhan. This paper selects that there are 30 cities in China transporting medical materials to Wuhan, and modeled it as 30 distribution centers and one demand point. This is the opposite of the traditional vehicle routing problem (VRP) with multiple demand points in one distribution center. In order to establish the model better, we assume that Wuhan is an emergency (distribution) center, 30 cities are regarded as customers waiting for distribution service, and the supply of medical materials in the city is regarded as the demand, which becomes a traditional vehicle routing problem. This paper first obtains the actual longitude and latitude of 30 cities and simulates the material demand of 30 cities. Then, using reverse thinking, it assumes that 30 cities (material supply points) are emergency material demand points, while Wuhan city is used as distribution warehouse for vehicle routing optimization. The distribution center (Wuhan) has a number of transport vehicles of the same type, which is responsible for medical supplies in 30 cities in turn distribution service, in order to ensure that all materials do not exceed the maximum load of the vehicle, through the reasonable optimization of the vehicle route, the total distribution distance is the smallest and the total cost is the lowest.

\subsection{Model assumptions}

The basic assumptions of the model are as follows:

- The speed of delivery vehicles is uniform and the same;

- The vehicle will return to the distribution center directly after completing the distribution task;

- There is only one distribution center (Wuhan), and the medical materials in the distribution center are sufficient to meet all needs;

- The demand of demand points (30 cities) is known and constant;

- All materials at each demand point can only be completed by one vehicle and cannot be distributed in batches;

- The delivery vehicles are trucks of the same model;

- The location and distance between each demand point and the distribution center are known.

\subsection{Modeling}

Based on the above assumptions, the emergency vehicle scheduling model is established with the minimum scheduling cost and distance as the goal. the mathematical model can be described as follows:

$$
\min c(x)=\sum_{k=1}^{K} \sum_{i=1}^{N} \sum_{j=1}^{N} c_{i j} x_{i j k}
$$

s.t.

$$
\sum_{k \in K} \sum_{j \in N} x_{i j k}=1, \forall i, j \in N
$$

$$
\begin{gathered}
\sum_{i \in N} \sum_{j \in N} d_{i} x_{i j k} \leq Q, \forall i, k \in K \\
\sum_{j \in N} x_{0 j k}=1, \forall k \in K \\
\sum_{i \in N} x_{i j k}-\sum_{j \in N} x_{j i k}=0, \forall k \in K, j \in N \\
\sum_{i \in N} x_{i j k}=1, \forall k \in K \\
x_{i j k}=\{0,1\}, \forall k \in K, \forall i, j \in N
\end{gathered}
$$

Where: $\mathrm{N}$ is the collection of service points and distribution centers; $\mathrm{i}$ and $\mathrm{j}$ respectively represent the number of the demand point; $\mathrm{K}$ is the total number of vehicles; $c_{i j}$ is the transportation cost of vehicles from $i$ to $\mathrm{j}$; $\mathrm{d}_{\mathrm{i}}$ is the demand of customer $\mathrm{i}$; $\mathrm{Q}$ is the maximum carrying capacity of the vehicle; $\mathrm{x}_{\mathrm{ijk}}$ is when vehicle $\mathrm{k}$ is assigned to run from customer $\mathrm{i}$ to customer $\mathrm{j}$, take 1 ; otherwise, take 0. Equation (1) represents the objective function is the lowest total distribution cost; Equation (2) indicates that each demand point can only be served by one vehicle once; equation (3) represents the capacity constraint of the vehicle; equation (4) indicates that the vehicle must start from the distribution center; equation (5) indicates that vehicle $\mathrm{k}$ must leave after the service of demand point $\mathrm{j}$ is completed; equation (6) indicates that each vehicle must return to the distribution center; equation (7) indicates that the constraint variable is $0-1$ variable.

\section{Emergency optimization \\ vehicle scheduling}

\subsection{Algorithm}

Genetic algorithm (GA) is an iterative search algorithm that simulates the process of biological evolution. The initial population is formed by coding. The chromosomes in the initial population are iterated through selection, crossover and mutation, and the search direction is adjusted adaptively. Finally, the chromosome with the highest fitness is obtained, that is, the optimal solution of the problem. Its basic idea is derived from Darwin's evolutionism "survival of the fittest, survival of the fittest". In 1975, John H.Holland In his monograph adaptation in natural and artificial systems, systematically discussed the overall framework of genetic algorithm, which laid a solid foundation for the wide application of genetic algorithm. Genetic algorithm has excellent robustness and potential parallel computing system, and has a great probability to find the optimal solution of the problem. Therefore, genetic algorithm has great advantages in solving CVRP problems.

In the iterative update process of the genetic algorithm, the fitness value of some individuals will be much larger than other individuals. These individuals will crowd out the poor individuals in the selection crossover and other genetic operations, resulting in reduced population diversity and easy to fall into local optimality. Simulated 
annealing introduces random factors in the search process, and accepts a solution that is worse than the current solution with a certain probability, which ensures the diversity of the population. Therefore, this paper chooses to combine the advantages of genetic algorithm and simulated annealing algorithm to avoid premature convergence of the algorithm and enhance the global search ability.

\section{2 vehicle routing problem with capacity}

The vehicle routing problem with capacity constraints can be described as: there is a distribution center with $M$ vehicles of the same model, and the maximum load of each vehicle is $\mathrm{Q}$. these vehicles start from the distribution center to serve $\mathrm{K}$ demand points, the demand of demand point $\mathrm{i}$ is $\mathrm{d}_{\mathrm{i}}$. Each demand point can only be visited by one vehicle, and each vehicle must return to the distribution center after service. The objective function is to find a vehicle distribution scheme that satisfies the constraint conditions, which can meet the needs of customers and minimize the total transportation distance and total transportation cost.

\section{Example Analysis}

This paper takes 30 cities in China as the material demand point, and Wuhan as the distribution center, on a computer with CPU of $2.60 \mathrm{GHz}$, memory of $2.59 \mathrm{ghz}$ and operating system of windows 10, simulated annealing-genetic algorithm (SA-GA) is verified by comparing the results of genetic algorithm. Suppose that there are 15 trucks in the distribution center, and the maximum load capacity of the trucks is 10 tons. The distribution center (Wuhan City) distributes emergency materials to 30 cities. The coordinates of the distribution center (Wuhan City) are (114.30, 30.60). The location coordinates and demand quantity of demand points are shown in Table 1. algorithm parameters: the maximum evolution algebra of the population is 800 , and the population size is 100

Table1. city coordinates and demand

\begin{tabular}{|c|c|c|c|c|c|c|c|}
\hline LABEL & DEMAND POINT & $\begin{array}{l}\text { LATITUDE AND } \\
\text { LONGITUDE }\end{array}$ & DEMAND & LABEL & DEMAND POINT & $\begin{array}{l}\text { LATITUDE AND } \\
\text { LONGITUDE }\end{array}$ & DEMAND \\
\hline 0 & WUHAN & $(114.30,30.60)$ & 0 & 16 & HEFEI & $(117.22,31.82)$ & 2 \\
\hline 1 & BEIJING & $(116.40,39.90)$ & 6 & 17 & NANJING & $(118.79,32.06)$ & 4 \\
\hline 2 & SHANGHAI & $(121.47,31.23)$ & 3 & 18 & HANGZHOU & $(120.20,30.25)$ & 2 \\
\hline 3 & FuZHOU & $(119.29,26.08)$ & 5 & 19 & NANCHANG & $(115.85,28.69)$ & 4 \\
\hline 4 & GUANGZHOU & $(113.26,23.13)$ & 4 & 20 & HAIKOU & $(110.31,20.04)$ & 3 \\
\hline 5 & JINAN & $(117.11,36.65)$ & 2 & 21 & NANNING & $(108.36,22.82)$ & 5 \\
\hline 6 & CHANGCHUN & $(125.32,43.81)$ & 4 & 22 & GUIYANG & $(106.63,26.65)$ & 4 \\
\hline 7 & HARBIN & $(126.53,45.80)$ & 5 & 23 & CHANGSHA & $(112.93,28.23)$ & 3 \\
\hline 8 & SHENYANG & $(123.46,41.68)$ & 3 & 24 & CHENGDU & $(104.07,30.65)$ & 2 \\
\hline 9 & НонНОт & $(111.74,40.84)$ & 2 & 25 & KUNMING & $(102.84,24.87)$ & 2 \\
\hline 10 & URUMQI & $(87.62,43.82)$ & 3 & 26 & LHASA & $(91.11,29.65)$ & 3 \\
\hline 11 & LANZHOU & $(103.83,36.06)$ & 4 & 27 & XINING & $(101.78,36.62)$ & 2 \\
\hline 12 & YINCHUAN & $(106.23,38.49)$ & 2 & 28 & TIANJIN & $(117.20,39.09)$ & 4 \\
\hline 13 & TAIYUAN & $(112.54,37.87)$ & 3 & 29 & CHONGQING & $(106.55,29.57)$ & 7 \\
\hline 14 & XIAN & $(108.94,34.34)$ & 6 & 30 & SHIJIAZHUANG & $(114.51,38.04)$ & 3 \\
\hline 15 & ZHENGZHOU & $(113.62,34.75)$ & 5 & & & & \\
\hline
\end{tabular}

\subsection{Case analysis}

In order to verify the superiority of simulated annealinggenetic algorithm, this article compares the traditional genetic algorithm to experiment. Among them, genetic algorithm uses a simulated annealing optimization strategy, and the remaining parameters are the same to ensure the fairness of the experiment. The experimental results are shown in the figure (1-2):

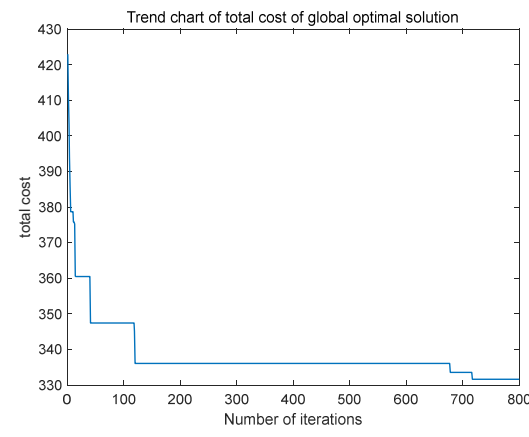

Fig 1. trend chart of SA-GA 


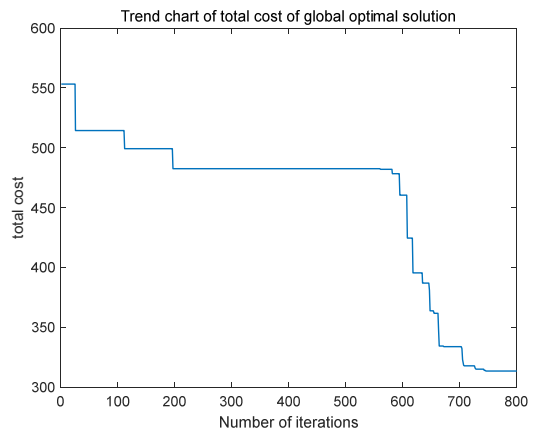

Fig 2. trend chart of GA

Comparing Figure 1 and Figure 2, it can be seen that the fitness function of Figure 1 has a faster convergence speed. In each program run, the running time of the algorithm is shorter, with an average of about 24 seconds, compared to the traditional genetic algorithm of figure 2 has obvious advantages in convergence speed. Although there is no big difference in the optimal path distance, the optimal path length of the traditional genetic algorithm is $331.6 \mathrm{~km}$, and the optimal path distance of the improved genetic algorithm is $313.6 \mathrm{~km}$, but the speed of the iteration to obtain the optimal solution is significantly increased. Therefore, it is of great help in solving the problem of emergency resource scheduling.

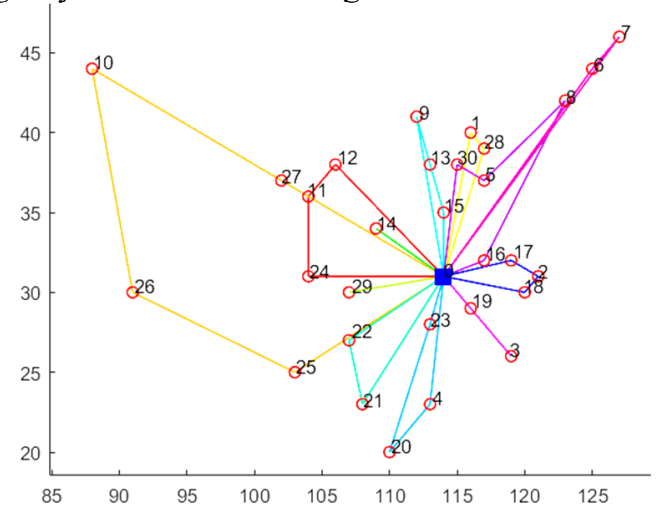

Fig 3. Global optimal route

It can be seen from Figure 3 that the total mileage of 12 vehicles is reduced, and the vehicle path is further optimized under the premise of meeting the maximum load constraint of the vehicle (10 tons), which shows that simulated annealing- genetic algorithm has good emergency resource scheduling Optimization ability. In the calculation example, the distribution schemes of the 12 vehicles are as follows (table2):

It can be seen from Table 2 that Route 1 is from Wuhan-Chengdu-Lanzhou-Yinchuan-Wuhan. When transporting materials from all parts of the country to Wuhan, a 10-ton truck can be sent from Chengdu or Yinchuan, and after passing through the other two cities, the materials were sent to Wuhan.

Table2. Optimal delivery route

\begin{tabular}{cc}
\hline Delivery route & Optimal delivery route \\
\hline 1 & $0-24-11-12-0$ \\
2 & $0-25-26-10-27-0$ \\
3 & $0-28-1-0$ \\
4 & $0-29-0$
\end{tabular}

\begin{tabular}{lc}
5 & $0-14-0$ \\
6 & $0-21-22-0$ \\
7 & $0-15-13-9-0$ \\
8 & $0-23-20-4-0$ \\
9 & $0-17-2-18-0$ \\
10 & $0-30-5-8-16-0$ \\
11 & $0-19-3-0$ \\
12 & $0-6-7-0$ \\
\hline
\end{tabular}

\section{Concluding remarks}

This paper proposes a genetic algorithm that improves group initialization and adopts simulated annealing optimization strategy. By accepting poor solutions with a certain probability to avoid falling into the local optimum, the local search ability of the genetic algorithm is greatly improved. Finally, through the example test of simulated annealing-genetic algorithm and the comparison with the optimal results of the traditional genetic algorithm, the stability and reliability of simulated annealing-genetic algorithm for solving the capacity-limited vehicle routing problem are verified. Considering the complexity of the problem, this paper only studies the uncertain factors under one vehicle type. In order to improve the practicality of the example model and the improved algorithm, the algorithm will be improved and applied to solve the multivehicle vehicle routing problem in the future. during the COVID-19 period, Wuhan is in urgent need of a large amount of medical supplies, and the optimization of emergency supplies scheduling can save time and transportation costs.

\section{Acknowledgment}

First of all, I would like to express my heartfelt thanks to my supervisor Xiang Xiaolin for his insightful guidance and careful advice on the research direction of my thesis, and for providing me with a large number of useful references. Secondly, I am also very grateful to my classmates for their direct and indirect help. During the process of writing the paper, we discussed and exchanged together, which made me actively encouraged in the process of writing this paper. Finally, I would like to thank the authors in the references. Their documents have given me more inspiration for writing the paper.

\section{References}

1. Chen Gang, Zhang Jin, Yan Xi. Agile distribution model of disaster relief emergency supplies [J]. Computer engineering and application, 2012,48 (34): 225-229.

2. Chen Gangtie, Shuai bin. Optimization of emergency vehicle scheduling based on fuzzy network and time dependence $[\mathrm{J}]$. Chinese Journal of safety Sciences, 2011, 21 (8): 171-176. 
3. Chen Sen, Jiang Jiang, Chen Ying Wu, et al. Model and application of vehicle distribution problem for emergency supplies in the case of undetermined road network structure. System engineering theory and practice, 2011, 31 (5): 907-913.

4. Yang Bin, Wen Hongrui, Li Feng, et al. Research on emergency material allocation decision under emergency scenario $[\mathrm{J}]$. Safety and environmental engineering, 2015,22 (5): 7-12.

5. Li Shuanglin, Ma zujun, Zheng bin, Dai Ying. Fuzzy multi-objective location and multimodal transportation problem for emergency materials distribution in the early stage after earthquake [J]. China management science, 2013,21 (02): 144-151.

6. Akhand M A H, Peya Z J, Sultana T, et al. Solving capacitated vehicle routing problem using variant sweep and swarm intelligence [J]. Journal of Applied Science and Engineering, 2017, 20 (4): 511-524.

7. J. H. Holland. Adaptation in natural and artificial Systems [M]. Ann Arbor: The University of Michigan Press, 1975:75.

8. Fiedrich F, Gehbauer F, Rickers U. Optimized Resource Allocation for Emergency Response after Earthquake Disaster[J].Safety Science, 2000, 35(1):41-57.

9. Chi H, Li J L, Shao X Y, et al. Timeliness Evaluation of Emergency Resource Scheduling[J].European Journal of Operational Research, 2017, 258(3):10221032.

10. Arun Jotshi, Gong, Rajan Batta. Dispatching and routing of emergency vehicles in disaster mitigation using data fusion. Socio-Economic Planning Sciences. 